A three-step classification framework to handle complex data distribution for radar UAV detection Jianfeng Ren, Xudong Jiang

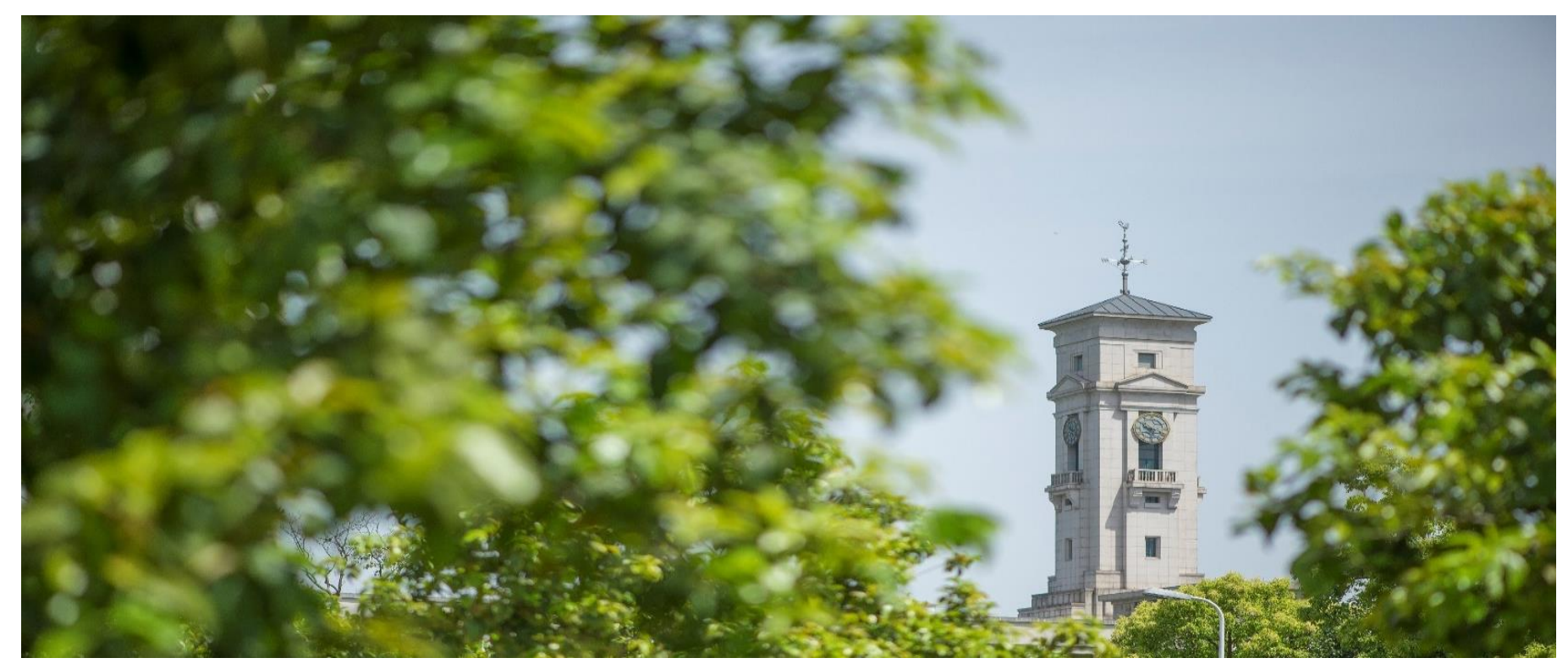


University of Nottingham Ningbo China, 199 Taikang East Road, Ningbo, 315100, Zhejiang, China.

First published 2020

This work is made available under the terms of the Creative Commons Attribution 4.0 International License:

http://creativecommons.org/licenses/by/4.0

The work is licenced to the University of Nottingham Ningbo China under the Global University Publication Licence:

https://www.nottingham.edu.cn/en/library/documents/research/global -university-publications-licence-2.0.pdf 


\title{
A Three-Step Classification Framework to Handle Complex Data Distribution for Radar UAV Detection
}

\author{
Jianfeng Ren* \\ The School of Computer Science, University of Nottingham Ningbo China, 199 Taikang \\ East Road, Ningbo, China, 315100. \\ Xudong Jiang \\ Electrical \& Electronic Engineering, Nanyang Technological University, Nanyang Link, \\ Singapore 639798.
}

\begin{abstract}
Unmanned aerial vehicles (UAVs) have been used in a wide range of applications and become an increasingly important radar target. To better model radar data and to tackle the curse of dimensionality, a three-step classification framework is proposed for UAV detection. First we propose to utilize the greedy subspace clustering to handle potential outliers and the complex sample distribution of radar data. Parameters of the resulting multi-Gaussian model, especially the covariance matrices, could not be reliably estimated due to insufficient training samples and the high dimensionality. Thus, in the second step, a multi-Gaussian subspace reliability analysis is proposed to handle the unreliable feature dimensions of these covariance matrices. To address the challenges of classifying samples using the complex multi-Gaussian model and to fuse the distances of a sample to different clusters at different dimensionalities, a subspace-fusion scheme is proposed in the third step. The proposed approach is validated on a large benchmark dataset, which significantly outperforms the state-of-the-art approaches.
\end{abstract}

Keywords: radar UAV detection, micro-Doppler signature, greedy subspace

\footnotetext{
*Corresponding author. Tel.: +86 (0)574 88180000 - 8805

Email addresses: jianfeng.ren@nottingham.edu.cn (Jianfeng Ren), exdjiang@ntu.edu.sg (Xudong Jiang)
} 
clustering, multi-Gaussian subspace reliability analysis, subspace fusion

\section{Introduction}

Unmanned aerial vehicles have become an increasingly important radar target because of the low cost, wide applications and potential threats to public security. According to Grand View Research [1], the global market for commercial UAVs will grow by $17 \%$ every year. UAVs have been used for many different applications, e.g., package delivery, land surveillance, traffic monitoring and chasing birds in airport. However, UAVs may impose threats to public security, e.g., UAVs near airport may jeopardize the safety of airplanes [2], or UAVs may carry bombs or dangerous chemicals in a terrorist attack. Thus, it has become increasingly important to reliably detect UAVs using radars.

Early techniques using kinematic and radar cross-section characteristics [3] could not reliably differentiate UAVs from birds, as the both kinds are small and slow-moving targets. Many representations of micro-Doppler signature (mDS) have been explored, e.g., spectrogram [4-11], cepstrogram [12], cadence velocity diagram [13-15], others [16-19], and combinations of the aforementioned [2022]. Particularly in [22], a rich source of features including spectrogram, cepstrogram and CVD are utilized. Most of the representations are closely related to spectrogram. mDS has been utilized in many radar-target-recognition tasks [23], e.g., airplane classification [24], ship detection [25], human detection [5], gait recognition [8, 9], action classification $[10,14]$ and vehicle classification [26]. Recently, Wi-Fi communication signals between UAVs and remote controllers have been utilized to detect UAVs [27]. However, when a UAV flies in an autonomous mode without Wi-Fi communication, such a technique will not work.

Machine-learning techniques have been utilized to automatically detect/classify UAVs using radars $[6,11,15,17-22,28]$. Artificial neural networks were applied on spectrum directly to classify different types of UAVs [28]. Support vector machine (SVM) and naive Bayes classifier were applied on the first five principal components extracted from spectrogram to differentiate UAVs from birds [6]. 
1 Huizing et al. employed Alexnet and LSTM-RNN on spectrograms to classify mini-UAVs [11], whereas Kim et al. utilized GoogLeNet on the image merged 3 from spectrogram and CVD [20]. Similarly, SVM was applied on the feature 4 vector obtained from spectrogram and CVD [21]. Patel et al. applied Alexnet 5 on four time-frequency representations including spectrogram, cepstrogram and - CVD for UAV classification [22]. Zhao and Su developed a cyclostationary analysis on the phase term of the radar signal to extract the mDS for UAV detection [18]. Very recently, empirical mode decomposition was employed to extract intrinsic mode functions for UAV classification [19]. Instead of detect10 ing/classifying one UAV at a time, Zhang and Li detected multiple UAVs by 11 using a k-means classifier on the mean CVD averaged along the Doppler fre12 quency [15]. Most of these approaches utilized spectrogram or time-frequency representations that are derived from spectrogram, e.g., cepstrogram and CVD. 14 Thus, the proposed approach also utilizes features derived from spectrogram. 15 However, most approaches utilized the magnitude spectrogram only. As shown 16 in [17], both phase and magnitude spectrograms are useful for classifying the 17 radar signal.

The authors recently developed an automated UAV-detection system utiliz19 ing the regularized 2-D complex-log Fourier transform to extract spectrogram20 like features and the subspace reliability analysis to remove unreliable feature ${ }_{21}$ dimensions [17]. Despite the success, three challenges remain. 1) The com22 plex sample distribution of radar data. Subspace approaches utilizing up to 23 the second-order statistics work well for Gaussianly distributed data [17, 29]. 24 However, the high-dimensional mDS features deviate largely from Gaussian. 2) 25 Outliers in radar data. Due to the poor signal-to-noise ratio of radar signal, 26 it is error-prone for human to label the data, which leads to mislabeled data ${ }_{27}$ (outliers). The outliers are harmful for training classifiers. 3) The curse of di28 mensionality. It is difficult to robustly model the complex data distribution in 29 a high-dimensional feature space.

In literature, these three challenges have been partially addressed. To model 31 the complex distribution of radar data, Regev et al. utilized artificial neural 
network to classify drones [28]. Zhao et al. utilized stacked auto-encoder and extreme learning machine for radar target recognition [30]. To be robust to the outliers in radar data, Dong et al. developed a joint sparse representation based on multi-task learning [31]. Many approaches have been devoted to address the curse of dimensionality. Specifically for radar target recognition, kernel joint discriminant analysis [32], sparse representation [31], subspace reliability analysis [17] and multiple kernel project subspace fusion [33] have been developed for dimensionality reduction. In this paper, an integrated three-step classification framework is proposed to address these three challenges.

In the first step, to handle the complex data distribution (Challenge 1) and the outliers (Challenge 2), the authors propose to utilize a greedy version of the sparse subspace clustering (SSC) algorithm [34, 35], the greedy subspace clustering (GSC) algorithm [36]. Gaussian mixture model (GMM) [37-39] is often used to model the complex data distribution, and the expectation-maximization (EM) algorithm [37] is often used to derive the mixture model. One critical challenge of the EM algorithm is that the GMM could not be reliably estimated due to insufficient training samples and the high feature dimensionality.

The sparse subspace clustering $[34,35]$ handles the complex distribution by clustering data according to the underlying subspace structure, which leads to a multi-Gaussian model if each cluster of samples follow the Gaussian distribution. The SSC algorithm is robust to outliers owing to the $l_{1}$ optimization when building the similarity matrix. As the SSC is slow, the authors propose to utilize the greedy subspace clustering [36]. Instead of the time-consuming $l_{1}$ optimization in the SSC, the GSC algorithm utilizes a nearest-subspace-neighbor algorithm to sequentially find the nearest neighbors to form linear subspaces. The neighborhood matrix is then used as the similarity matrix for subsequent spectral clustering. Similar outliers may form a cluster. Thus, a drop-off technique is proposed to remove samples in the smallest cluster as outliers.

In the second step, to tackle the curse of dimensionality (Challenge 3), a multi-Gaussian subspace reliability analysis (MGSRA) is proposed to remove the unreliable feature dimensions of the multi-Gaussian model derived in the first 
1 step. The model cannot be reliably estimated due to insufficient samples in each cluster and the high dimensionality, especially the dimensions corresponding to

3 the small eigenvalues of covariance matrices. As the inverse of covariance matrix

4 is used to weigh the feature dimensions, those small eigenvalues will impose very

5 large and problematic weights to the corresponding dimensions [17, 29, 40].

6 Thus, the MGSRA algorithm is proposed to handle those unreliable feature

7 dimensions separately at different subspaces.

8 The proposed MGSRA is different from previous approaches $[17,41]$ in the 9 following aspects: 1) Most subspace approaches are designed based on a uni10 Gaussian model, whereas the MGSRA is built on a multi-Gaussian model, which 11 could better model the distribution of radar data. 2) Most subspace approaches 12 aim to find one linear subspace that meets a certain optimization criterion, 13 whereas the proposed MGSRA aims to find a set of linear subspaces separately 14 for each class. A problem thus arises naturally: how to optimally combine the 15 results from different subspaces?

In the third step, a subspace-fusion scheme is proposed to combine these 17 results. More specifically, the Mahalanobis distances of a sample to each cluster 18 center at a set of given feature dimensionalities are calculated. The rational of choosing multiple dimensionalities is that it is difficult to determine the optimal feature dimensionality for subspace approaches. Thus, a range of dimension${ }_{21}$ alities covering the optimal one are sampled and the Mahalanobis distances at 22 these dimensionalities are evaluated. Then, the distances of a sample to different cluster centers of different classes at different subspace dimensionalities are treated as a feature vector, and a support vector machine is trained to combine

25 these distances. The proposed subspace fusion works better than traditional

26 approaches in which the distances are merged as a posterior probability, and 27 evaluated only at some fixed dimensionality for each class [37, 38].

28 The contributions of this study are summarized as follow: 1) Three chal29 lenges for radar UAV detection are identified: the complex data distribution, 30 the outliers and the curse of dimensionality. 2) A three-step classification frame31 work is proposed to address these challenges, i.e. a) the greedy subspace clus- 
1 tering is utilized to handle the complex distribution and the outliers of radar

2 data; b) a multi-Gaussian subspace reliability analysis is proposed to tackle the

3 unreliable feature dimensions of the derived model; c) a subspace-fusion scheme

4 is proposed to combine the subspace distances. 3) The proposed approach is

5 systematically evaluated on a large benchmark dataset, and demonstrates a

6 superior performance compared with the state-of-the-art approaches.

\section{2. Proposed Three-Step Classification Framework}

\section{2.1. Challenges of UAV Detection}

There are many challenges in detecting/classifying UAVs. Two of them, a robust feature representation and unreliable feature dimensions, were well addressed in the previous work [17]. Three others remain: the complex data distribution, the outliers and the curse of dimensionality.

\subsubsection{Complex Sample Distribution of Radar Data}

Subspace approaches often assume that data follow the Gaussian distribution [17, 29, 40-44], as the Gaussian model can be built using only mean and variance (covariance for multivariate Gaussian), which can be estimated easily from the data. However, in [29], Ren et al. showed that for visual recognition the histogram-like features do not follow the Gaussian distribution.

In this study, the authors find that it is insufficient to use a Gaussian distribution to model either the UAV class or the non-UAV class, as shown in Fig. 3 later in Section 7.2. This is primarily due to the following: 1) There are many different types of UAVs, e.g., helicopter, tricopter, quadcopter, hexacopter, octocopter and fixed-wing plane. One Gaussian distribution is not sufficient to model all these UAVs, especially the fixed-wing plane is significantly different from the rest. 2) The non-UAV class cannot be modeled as one Gaussian model either, as it consists of distinct background samples and bird samples. 3) Even for the same type of UAVs, data may not be Gaussianly distributed. All these lead to a complex data distribution. 


\subsubsection{Outliers in Radar Data}

The radar micro-Doppler signatures are weak, much weaker than the main body Doppler. In addition, the thermal noise in a circuit and the noise/interference to radar receiver may contaminate radar signals. All these make it difficult to label radar targets. The labeling errors may come from different sources: 1) As the micro-Doppler signatures are weak, it is error-prone to manually label the data by analyzing the radar recordings; 2) The radar may capture the micro-motions of background objects, which will distort the radar signals of the target; 3) Due to the narrow radar beam, the target may fly in and out of the radar beam irregularly, without the notice of operators. 4) The mDS of fixedwing plane is similar to that of gliding birds, which may be wrongly labeled as non-UAV if the UAV flies too far away and is not clearly visible in the video recordings. Some radar targets thus may be wrongly labeled, and known as outliers.

\subsubsection{Curse of Dimensionality}

The curse of dimensionality arises mainly due to high feature dimensionality and insufficient data. The feature representation used in the proposed approach, regularized 2-D complex-log Fourier transform [17], leads to a high-dimensional feature vector. (Refer to Table 7 for more information.) As a result, it is difficult to precisely model the complex data distribution using the limited number of samples in such a high-dimensional feature space.

More specifically, the high dimensionality leads to the following: 1) Traditional approaches such as the EM algorithm for GMM [37] do not work well here. The Gaussian mixture models are often either over-simplified or poorly estimated. The authors thus propose to utilize the greedy subspace clustering to find the underlying linear subspaces. 2) After clustering, it is still difficult to reliably estimate the multi-Gaussian model due to the high dimensionality, especially the dimensions corresponding to the small eigenvalues of the covariance matrices. Thus, a MGSRA algorithm is proposed to tackle these unreliable dimensions. 3) It is difficult to use the derived multi-Gaussian model to classify 
samples in the high-dimensional feature space. Therefore, the authors propose

2 to evaluate the Mahalanobis distances in the reduced subspaces at multiple

dimensionalities and fuse them using the proposed subspace-fusion scheme.

\section{2.2. Overview of the Proposed Approach}

5 The block diagram of the proposed approach is shown in Fig. 1. The initial 6 features are extracted by using the robust spectral analysis [17], and then the proposed three-step classification framework shown in Fig. 1 tackles the afore8 mentioned three challenges. 1) To tackle the curse of dimensionality, among 9 various clustering algorithms, the authors propose to utilize the greedy sub10 space clustering, as it could model the complex data distribution and handle 11 the outliers at the same time. 2) The covariance matrices of the derived multi-

12 Gaussian model are important but difficult to be reliably estimated. Thus, a 13 multi-Gaussian subspace reliability analysis is proposed to tackle the unreliable 14 feature dimensions of the covariance matrices. 3) Finally, a subspace-fusion 15 scheme is proposed to evaluate the Mahalanobis distances of a sample to mul-

16 tiple cluster centers at different subspace dimensionalities. These distances are then fused by a support vector machine. In the following sections, the proposed 18 approach will be illustrated in details.

\section{Robust Spectral Analysis}

The initial features are extracted using the regularized 2D complex-logFourier transform in [17]. The procedures are briefly summarized as follow:

22 Firstly, the time-series radar signal $s(t)$ is segmented into $I$ overlapping 23 frames $\left\{s_{0}, s_{1}, \ldots, s_{I-1}\right\}$, where $s_{i}=\left\{s_{i}[n], n=0,1, \ldots, J-1\right\}$ is a vector ${ }_{24}$ of length $J$. These $I$ frames form a synthetic image $\boldsymbol{S}=\left[\boldsymbol{s}_{0}, \boldsymbol{s}_{1}, \ldots, \boldsymbol{s}_{I-1}\right]$ of 25 size $I \times J$. The discrete Fourier transform $\boldsymbol{f}_{i}=\left[f_{i, 0}, f_{i, 1}, \ldots, f_{i, J-1}\right]$ of $\boldsymbol{s}_{i}$, ${ }_{26} \boldsymbol{f}_{i}=\mathcal{F}\left\{\boldsymbol{s}_{i}\right\}$, is computed as:

$$
f_{i, k}=\sum_{n=0}^{J-1} s_{i}[n] \exp \left\{-j 2 \pi \frac{k n}{J}\right\}, k=0,1, \ldots, J-1,
$$




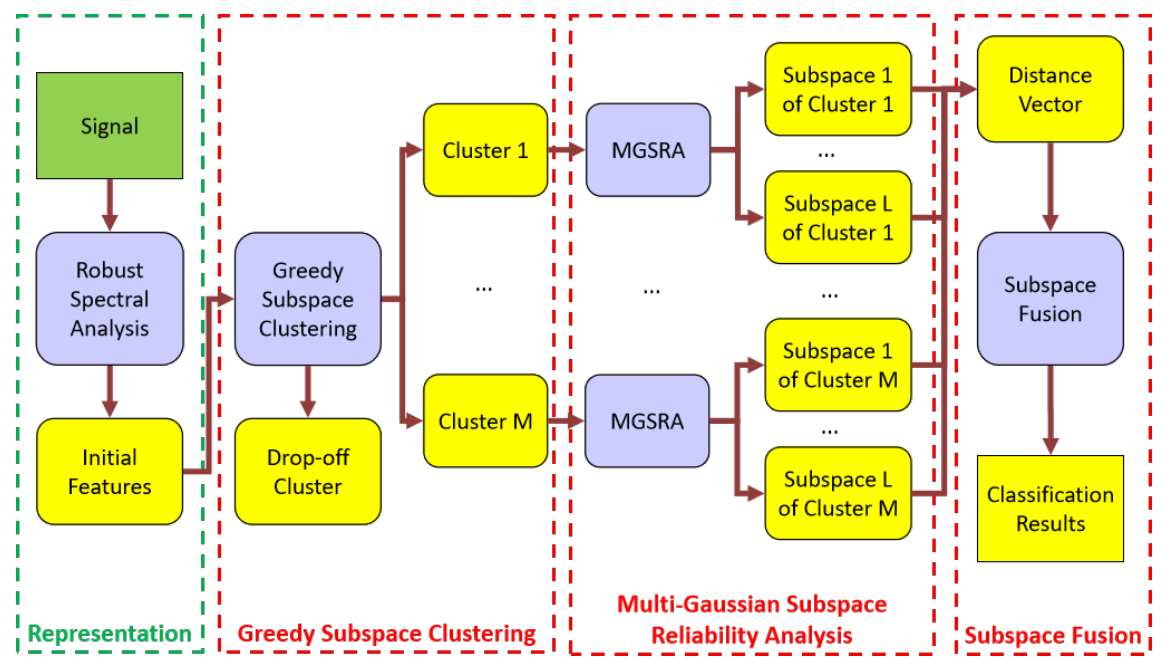

Figure 1: The proposed three-step classification framework shown in the three red boxes.

1 where $\mathcal{F}\{\cdot\}$ denotes the discrete Fourier transform.

Secondly, the 2-D complex Fourier transform of $\boldsymbol{S}$ is derived, which is equivalent to two 1-D spectral analysis on $\boldsymbol{S}$ :

$$
\mathcal{F}_{2 D}\{\boldsymbol{S}\}=\mathcal{F}_{t}\{\mathcal{F}\{\boldsymbol{S}\}\}
$$

where $\mathcal{F}\{\boldsymbol{S}\}$ is the Fourier transform on $\boldsymbol{S}$, and $\mathcal{F}_{t}\{\cdot\}$ is the Fourier transform along the time axis. Previous approaches $[12,13]$ often utilize only the mag8 nitude of $\mathcal{F}\{\boldsymbol{S}\}$, whereas both phase and magnitude of $\mathcal{F}\{\boldsymbol{S}\}$ are utilized in

${ }_{9} \mathcal{F}_{t}\{\cdot\}$, because phase spectrums also carry important discriminant information 10 for classification [17].

11 Thirdly, the weak micro-Doppler signatures are enhanced by taking the log12 arithm of the spectrum [17]. For $\boldsymbol{f}_{i}=\mathcal{F}\left\{\boldsymbol{s}_{i}\right\}=\boldsymbol{m}_{i} \exp \left\{j \boldsymbol{\theta}_{i}\right\}$,

$$
\log \left\{\boldsymbol{f}_{i}\right\}=\log \left\{\boldsymbol{m}_{i}\right\}+j \boldsymbol{\theta}_{i}
$$

15 where $\boldsymbol{m}_{i}$ is the magnitude spectrum and $\boldsymbol{\theta}_{i}$ is the phase spectrum. To balance

16 the effects of $\log \left\{\boldsymbol{m}_{i}\right\}$ and $\boldsymbol{\theta}_{i}$, a weighting factor $w$ is introduced:

$$
\log \left\{\boldsymbol{f}_{i}\right\}=\log \left\{\boldsymbol{m}_{i}\right\}+j w \boldsymbol{\theta}_{i} .
$$


$1 \quad w$ is simply set as $w=1 / \pi$ so that the phase term is normalized to $[-1,1]$.

Fourthly, a regularization term is introduced to Eqn. (4) to reduce the noise, 3 because taking the logarithm not only enhances the weak micro-Doppler signa4 ture, but also enlarges the noise.

$$
\log \left\{\boldsymbol{f}_{i}\right\}=\log \left\{\boldsymbol{m}_{i}+c_{i}\right\}+j w \boldsymbol{\theta}_{i},
$$

where $c_{i}=\operatorname{med}\left\{\boldsymbol{m}_{i}\right\}$, i.e. the median value of $\boldsymbol{m}_{i}$. By adding such a regulariza-

8 tion constant $c_{i}$, the logarithm of the strong frequency component will not be

9 significantly altered, whereas the logarithm of the weak frequency component will be regularized close to $\log c_{i}$. The variations of noise frequency components

11 are hence greatly reduced. Readers may refer to [17] for more details on the 12 noise robustness of the robust spectral analysis.

13 Finally, the regularized 2-D complex-log-Fourier transform is derived as:

$$
\mathcal{F}_{R}\{\boldsymbol{S}\}=\mathcal{F}_{t}\{\log \{\mathcal{F}\{\boldsymbol{S}\}\}\}
$$

16 where $\log \{\mathcal{F}\{\boldsymbol{S}\}\}$ is calculated according to Eqn. (5).

\section{4. Greedy Subspace Clustering}

\subsection{Limitations of EM Algorithm for Gaussian Mixture Model}

The Gaussian mixture models $[37,38]$ have been widely used to handle complex data distributions. For $D$-dimensional feature $\boldsymbol{x} \in \mathbb{R}^{D}$, the mixture probability density function (PDF) of the likelihood function is defined as:

$$
p(\boldsymbol{x} \mid \boldsymbol{\Theta})=\sum_{i=1}^{M} \alpha_{i} p_{i}(\boldsymbol{x}) .
$$

This PDF is a weighted linear combination of $M$ Gaussian densities $p_{i}(\boldsymbol{x})$, each parameterized by a mean vector $\boldsymbol{\mu}_{i} \in \mathbb{R}^{D}$ and a covariance matrix $\boldsymbol{\Sigma}_{i} \in \mathbb{R}^{D \times D}$,

$$
p_{i}(\boldsymbol{x})=\frac{1}{(2 \pi)^{D / 2}\left|\Sigma_{i}\right|^{1 / 2}} \exp \left\{-\frac{1}{2}\left(\boldsymbol{x}-\boldsymbol{\mu}_{i}\right)^{T} \boldsymbol{\Sigma}_{i}^{-1}\left(\boldsymbol{x}-\boldsymbol{\mu}_{i}\right)\right\} .
$$

Collectively, the model is denoted as $\boldsymbol{\Theta}=\left\{\alpha_{i}, \boldsymbol{\mu}_{i}, \boldsymbol{\Sigma}_{i}\right\}$, for $i=1,2, \ldots, M$. 
28 is a vector whose nonzero entries correspond to the points in $\boldsymbol{Y}_{\hat{i}}$ lying in the

29 same subspace as $\boldsymbol{y}_{\boldsymbol{i}}$. By inserting a zero entry at the $i$-th row of $\boldsymbol{c}_{i}$, it becomes for each sample; 2) Estimate the cluster weight, the mean and the covariance matrix of each cluster. Due to the curse of dimensionality, it is difficult to use the EM algorithm to build a precise mixture model for radar data. Hence the model is often simplified, e.g., by sharing the same covariance matrix among different classes and/or different mixture components, or by assuming that the covariance matrix is diagonal [37]. All these may oversimplify the model so that the discrimination power of the GMM is greatly reduced. To address this problem, the authors propose to utilize the greedy subspace clustering [36].

\subsection{Motivations of Greedy Subspace Clustering}

The greedy subspace clustering [36] is a greedy version of the sparse subspace clustering [35, 45]. The subspace clustering problem is formally defined as: Given data points $\left\{\boldsymbol{y}_{i} \in \mathbb{R}^{D}\right\}_{i=1}^{N}$ drawn from a union of independent linear subspaces $\left\{\mathcal{S}_{i}\right\}_{i=1}^{n}$, the target is to find dimensions $\left\{d_{i}\right\}_{i=1}^{n}$, subspace bases $\left\{\boldsymbol{A}_{i} \in \mathbb{R}^{D \times d_{i}}\right\}_{i=1}^{n}$ and permutation matrix $\boldsymbol{\Gamma} \in \mathbb{R}^{N \times N}$ that segment the data,

$\boldsymbol{Y}=\left[\boldsymbol{y}_{1}, \boldsymbol{y}_{2}, \ldots, \boldsymbol{y}_{N}\right]=\left[\boldsymbol{Y}_{1}, \boldsymbol{Y}_{2}, \ldots, \boldsymbol{Y}_{n}\right] \boldsymbol{\Gamma}$,

where $\boldsymbol{Y}_{i} \in \mathbb{R}^{D \times N_{i}}$ are $N_{i}$ data points drawn from $\mathcal{S}_{i}$ and $N=\sum_{i=1}^{n} N_{i}$. If $\boldsymbol{y}$ is a new data point in $\mathcal{S}_{i}$, it can be represented as a linear combination of the $d_{i}$ points in the same subspace.

Let $\boldsymbol{Y}_{\hat{i}} \in \mathbb{R}^{D \times(N-1)}$ denote the matrix obtained from $\boldsymbol{Y}$ by removing the $i$-th column $\boldsymbol{y}_{i}$, where $\hat{i}$ means "not $i$ ". $\boldsymbol{c}_{i} \in \mathbb{R}^{N-1}$ derived by solving the $l_{1}$ optimization problem,

$$
\operatorname{argmin}_{\boldsymbol{c}_{i}}\left\|\boldsymbol{c}_{i}\right\|_{1} \text { subject to } \boldsymbol{y}_{\boldsymbol{i}}=\boldsymbol{Y}_{\hat{i}} \boldsymbol{c}_{i}
$$

The Expectation-Maximization algorithm [37] is often used to derive the mixture model. It starts with some initial estimation of $\boldsymbol{\Theta}$, and then updates $\Theta$ by iteratively altering the following: 1) Estimate the membership weights 
$1 \quad$ an $N$-dimensional vector $\tilde{\boldsymbol{c}}_{i} \in \mathbb{R}^{N}$. The $l_{1}$ optimization is repeated for every $\boldsymbol{y}_{i}$, $2 i=1,2, \ldots, N$. Then, the following coefficient matrix is obtained:

$$
\boldsymbol{C}=\left[\tilde{\boldsymbol{c}}_{1}, \tilde{\boldsymbol{c}}_{2}, \ldots, \tilde{\boldsymbol{c}}_{N}\right] \in \mathbb{R}^{N \times N},
$$

5 which can be seen as the similarity matrix for $\boldsymbol{Y}$. Then, the spectral clustering 6 algorithm [36] is applied on $\boldsymbol{C}$ to segment the data.

The sparse subspace clustering is robust to noise and outliers owning to the

${ }_{8} l_{1}$ optimization, but $l_{1}$ optimization is slow. On the collected UAV-detection 9 dataset consisting of more than 10,000 training samples of 7236 dimensions, 10 it takes more than 300 seconds for the $l_{1}$ optimization of one sample. The 11 total execution time for all samples is about 35 days, which is too long. In 12 addition, memory of a few gigabytes is required for each $l_{1}$ optimization, and ${ }_{13}$ hence parallel computing using a graphic card is not a feasible option. These 14 are the motivations of using the greedy subspace clustering [36].

\section{4.3. Nearest-Subspace-Neighbor Algorithm}

The greedy subspace clustering [36] utilizes a nearest-subspace-neighbor (N-

${ }_{17} \mathrm{SN}$ ) algorithm to sequentially find nearest neighbors to the subspace spanned by 18 point $\boldsymbol{y}_{i}$ and existing neighbors. The spectral clustering algorithm [36] is then 19 applied on the neighborhood matrix for clustering. Formally, let $\mathcal{I}_{i}$ denote the 20 set of neighbors for data point $\boldsymbol{y}_{i},[N]$ denote the set $\{1,2, \ldots, N\}, \mathcal{U}$ denote the ${ }_{21}$ subspace spanned by the set of neighbors $\mathcal{I}_{i}, \boldsymbol{U}$ denote the set of orthonormal 22 bases of subspace $\mathcal{U}$, and $\mathbb{I}\{\cdot\}$ denote an indicator function, which is one if the 23 statement is true and zero otherwise. The NSN algorithm is summarized in 24 Algorithm 1.

By initializing $\mathcal{I}_{i} \leftarrow\{i\}$, the first neighbor is chosen as $\boldsymbol{y}_{i}$. The NSN algo26 rithm then finds $K$ neighbors sequentially. At step $k$, a $k$-dimensional subspace ${ }_{27} \mathcal{U}$ spanned by $\boldsymbol{y}_{i}$ and the $k-1$ neighbors is constructed, and the point closest ${ }_{28}$ to the subspace is selected. After $k>k_{\max }$, the subspace $\mathcal{U}$ constructed at step ${ }_{29} k_{\max }$ is used, and the points closest to the subspace $\mathcal{U}$ are chosen as neighbors 30 for the rest of the process. 


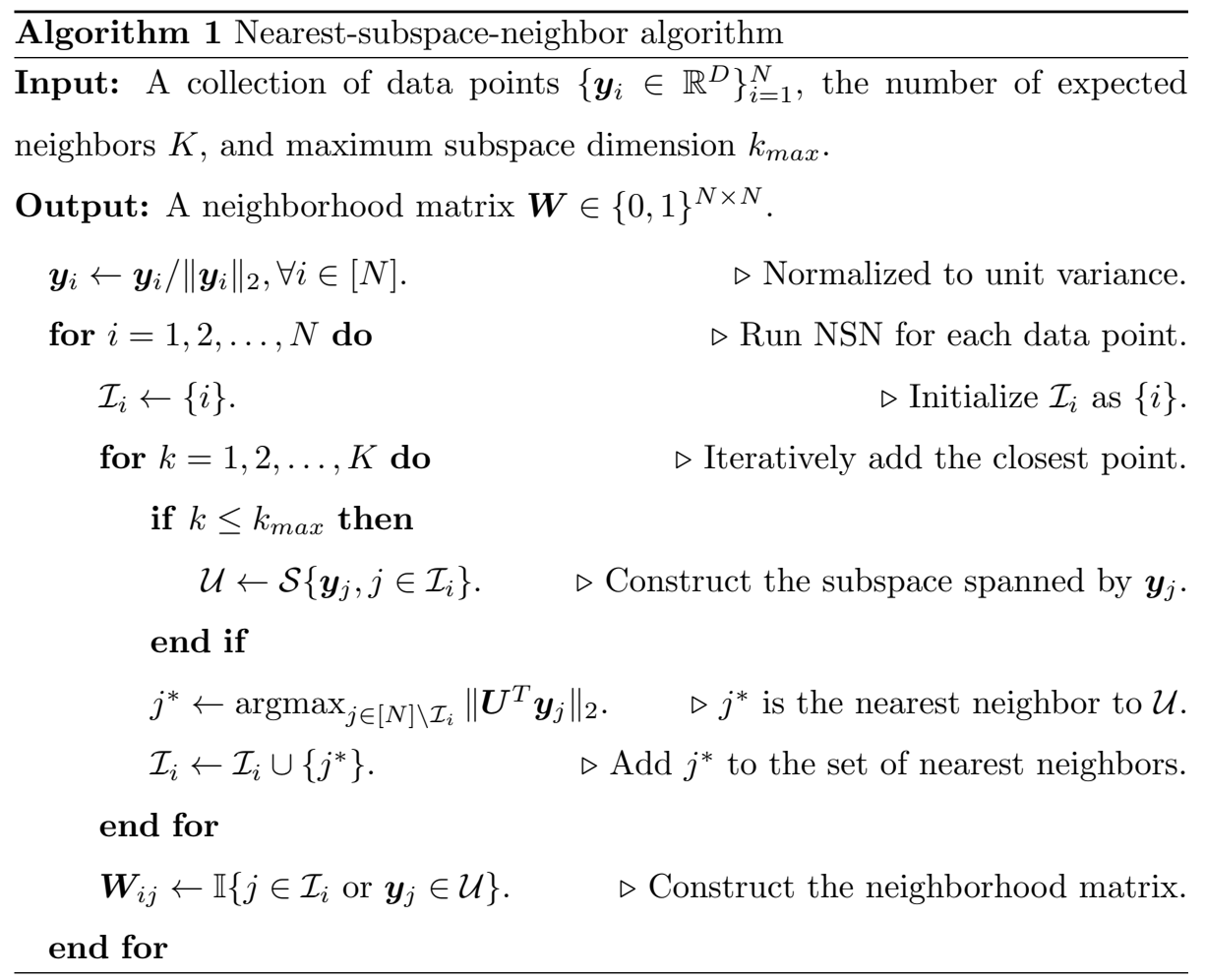


A brief time complexity analysis of the NSN algorithm is presented as fol2 low: At step $k$ of the inner loop, the time complexity is $O\left(D^{2}+k^{3}\right)$ to derive 3 the spanned subspace $\mathcal{U}$ using Singular Value Decomposition. The time for ${ }_{4} \operatorname{argmax}_{j \in[N] \backslash \mathcal{I}_{i}}\left\|\boldsymbol{U}^{T} \boldsymbol{y}_{j}\right\|_{2}$ is $O(k D N)$. Thus, the time for the most inner loop in 5 Algorithm 1 is $O\left(D^{2}+k^{3}+k D N\right)$. The time complexity for the whole algorithm 6 is $O\left(N\left(K D^{2}+\sum_{k=1}^{K} k^{3}+\sum_{k=1}^{K} k D N\right)=O\left(N K D^{2}+N K^{4}+N D K^{2}\right)\right.$. Note 7 that when $K \ll D$, the time complexity can be simplified as $O\left(N K D^{2}\right)$.

8 The GSC algorithm clusters data according to the underlying subspace struc9 ture. As a result, a multi-Gaussian model is derived for each class. The pro10 posed MGSRA algorithm then removes the unreliable feature dimensions of the ${ }_{11}$ derived model, as illustrated in Section 5.

12 4.4. Outlier Removal by Cluster Drop-off

Although the GSC algorithm is robust to outliers to some extent, mislabeled 14 data may be similar to each other and form a cluster, e.g., the UAV may fly 15 out of the sight of a radar, but the radar recordings may be mislabeled as ${ }_{16}$ UAV samples, and these similar outliers may form a cluster. To tackle this 17 problem, a simple heuristic is proposed to remove the outliers, i.e., the cluster 18 with the smallest number of samples for each class is dropped off. In general, 19 the mislabeled data only account for a small portion of the dataset. If they form 20 a cluster, most likely they form the smallest cluster.

\section{5. Multi-Gaussian Subspace Reliability Analysis}

In [17], the subspace reliability analysis was utilized to remove the unreliable feature dimensions in the UAV and non-UAV classes separately in two different subspaces. The samples of each class are assumed to follow the Gaussian distribution. However, one Gaussian is not sufficient to model the complex data distribution. The authors thus propose to utilize the greedy subspace clustering to find the underlying subspace structure, as shown in the previous section, which naturally leads to a multi-Gaussian model. 
3 model is given as:

The derived model could not be reliably estimated due to the curse of di-

$$
p_{i j}(\boldsymbol{x})=\frac{1}{(2 \pi)^{D / 2}\left|\sum_{i j}\right|^{1 / 2}} \exp \left\{-\frac{1}{2}\left(\boldsymbol{x}-\boldsymbol{\mu}_{i j}\right)^{T} \boldsymbol{\Sigma}_{i j}^{-1}\left(\boldsymbol{x}-\boldsymbol{\mu}_{i j}\right)\right\},
$$

where $\boldsymbol{\mu}_{i j} \in \mathbb{R}^{D}$ and $\boldsymbol{\Sigma}_{i j} \in \mathbb{R}^{D \times D}$ are the mean vector and the covariance matrix for the $i$-th cluster of the $j$-th class, respectively. The key issue here is to reliably estimate the covariance matrices $\boldsymbol{\Sigma}_{i j} \in \mathbb{R}^{D \times D}$ so that the Mahalanobis distance $\left(\boldsymbol{x}-\boldsymbol{\mu}_{i j}\right)^{T} \boldsymbol{\Sigma}_{i j}^{-1}\left(\boldsymbol{x}-\boldsymbol{\mu}_{i j}\right)$ could be evaluated reliably. The small eigenvalues of the covariance matrices could not be reliably estimated. As the inverse of $\boldsymbol{\Sigma}_{i j}$ is used to weigh the feature dimensions, those small eigenvalues are harmful for classification [40]. If the number of samples $N<D$, some eigenvalues of $\boldsymbol{\Sigma}_{i j}$ will be zero and induce infinitely large weights. Even in the case that $\boldsymbol{\Sigma}_{i j}$ has full rank, the small eigenvalues of $\boldsymbol{\Sigma}_{i j}$ still cause trouble, as their inverses introduce problematic large weights to the feature dimensions. To tackle this problem, a multi-Gaussian subspace reliability analysis is proposed.

Denote the Mahalanobis distance of $\boldsymbol{x}$ to the $i$-th cluster of the $j$-th class as

$$
d_{i j}(\boldsymbol{x})=\frac{1}{2}\left(\boldsymbol{x}-\boldsymbol{\mu}_{i j}\right)^{T} \boldsymbol{\Sigma}_{i j}^{-1}\left(\boldsymbol{x}-\boldsymbol{\mu}_{i j}\right) .
$$

The targets are to remove the small eigenvalues of $\boldsymbol{\Sigma}_{i j}$ so that $d_{i j}(\boldsymbol{x})$ could be evaluated reliably, and to preserve the discriminant information among different classes, which mainly resides in the between-class scatter matrix

$$
\boldsymbol{\Sigma}_{b}=\sum_{j=1}^{c}\left(\boldsymbol{\mu}_{j}-\boldsymbol{\mu}\right)\left(\boldsymbol{\mu}_{j}-\boldsymbol{\mu}\right)^{T},
$$

where $\boldsymbol{\mu}_{j}$ is the mean vector for the $j$-th class, $\boldsymbol{\mu}$ is the global mean and the number of class $c=2$ for UAV detection. To remove the unreliable feature dimensions of $\boldsymbol{\Sigma}_{i j}$, and preserve the discriminant information in $\boldsymbol{\Sigma}_{b}$, the eigendecomposition is applied on $\boldsymbol{S}_{i j}=\boldsymbol{\Sigma}_{i j}+\boldsymbol{\Sigma}_{b}$ as:

$$
\boldsymbol{S}_{i j}=\boldsymbol{\Phi}_{i j} \boldsymbol{\Lambda}_{i j} \boldsymbol{\Phi}_{i j}^{T}
$$


1 where $\boldsymbol{\Phi}_{i j}$ and $\boldsymbol{\Lambda}_{i j}$ are the eigenvector and eigenvalue matrices of $\boldsymbol{S}_{i j}$, respective-

2 ly. Then, the eigenvectors are chosen corresponding to the leading $m$ eigenvalues

${ }_{3}$ of $\boldsymbol{S}_{i j}$, i.e., $\boldsymbol{\Phi}_{i j m}$, as the projection matrix. The Mahalanobis distance $d_{i j}(\boldsymbol{x})$ in

4 the projected $m$-dimensional subspace becomes:

$$
d_{i j m}(\boldsymbol{x})=\left(\boldsymbol{x}-\boldsymbol{\mu}_{i j}\right)^{T} \boldsymbol{\Phi}_{i j m}\left(\boldsymbol{\Phi}_{i j m}^{T} \boldsymbol{\Sigma}_{i j} \boldsymbol{\Phi}_{i j m}\right)^{-1} \boldsymbol{\Phi}_{i j m}^{T}\left(\boldsymbol{x}-\boldsymbol{\mu}_{i j}\right)
$$

The optimal feature dimensionality cannot be easily determined. Thus, 8 many subspace approaches report the classification accuracies at different di9 mensionalities to show how the accuracies vary with the dimensionality, without determining the optimal dimensionality in advance. In the proposed approach, 1 the distances are evaluated at a range of dimensionalities that probably will 12 cover the optimal one. As these distances are evaluated in different subspaces, 13 their scalings are different, and they should be properly weighted before fusion.

14 Most importantly, a proper classification scheme needs to be developed for the 15 derived multi-Gaussian model. To address these challenges, a subspace-fusion 16 scheme is proposed as illustrated in the next section.

\section{Proposed Subspace-Fusion Scheme}

The proposed subspace-fusion scheme aims to combine the Mahalanobis distances defined in Eq. (16) and to build a classifier for the derived multi-Gaussian model. These Mahalanobis distances form a feature vector,

$$
\boldsymbol{d}\left(\boldsymbol{x}_{k}\right)=\left[d_{i j m}\left(\boldsymbol{x}_{k}\right)\right]
$$

23 where $i=1,2, \ldots, M_{j}$ is the index of clusters, $j=1,2, \ldots, c$ is the index of 24 classes, and $m$ is the index of subspace dimensionalities. Assume that all class25 es have the same number of clusters $M$, and the Mahalanobis distances are 26 evaluated at $L$ different subspace dimensionalities for each cluster, the distance ${ }_{27}$ vector $\boldsymbol{d}\left(\boldsymbol{x}_{k}\right) \in \mathbb{R}^{M c L}$. A support vector machine is trained using the derived ${ }_{28}$ feature vector $\boldsymbol{d}\left(\boldsymbol{x}_{k}\right), k=1,2, \ldots, N$. For a new testing sample $\boldsymbol{t}, \boldsymbol{d}(\boldsymbol{t})$ is e29 valuated and the class label is predicted using the trained SVM. The proposed so subspace-fusion scheme has the following advantages: 
1. For many subspace approaches $[17,29,40-44]$, it is difficult to find the optimal feature dimensionality. In the proposed approach, the Mahalanobis distances are evaluated at a given set of dimensionalities, without the need of selecting the optimal dimensionality. It is much easier to choose a range of dimensionalities covering the optimal one, than to precisely determine it in advance.

2. The proposed scheme determines the optimal weights for the Mahalanobis distances through the trained SVM, which solves the problem of optimally combining these distances. It definitely outperforms other approaches in which the distances are evaluated at a single (optimal) dimensionality [37].

3. The proposed scheme addresses the challenge of developing a proper classifier for the multi-Gaussian model. Traditional maximum-a-posterior classifier for the GMM [37] cannot work properly here as the posterior probabilities cannot be reliably estimated due to the curse of dimensionality. The proposed approach tackles the problem by evaluating the distances in many reduced subspaces, and fusing them using a support vector machine.

\section{Experimental Evaluation}

\subsection{Experimental Setup}

The measurement data were collected by Thales using a low-power continuouswave radar operating at $\mathrm{X}$-band $(9.7 \mathrm{GHz}$ radio frequency). Some signals were sampled at $192 \mathrm{kHz}$ and others at $96 \mathrm{kHz}$. They are all normalized to $96 \mathrm{kHz}$ in the experiments. A horn antenna was manually adjusted toward the nearby target object. Bird samples were collected within the distance of 5-50 meters to the radar, and UAV samples were collected within the distance of 3-150 meters to the radar.

The dataset used in [17] consists of multiple radar recordings of UAVs and birds, varying in length. The total length of all recordings reaches 1058 seconds, including 854 seconds of UAV recordings and 204 seconds of non-UAV recordings. To better evaluate the performance of the proposed system, the dataset 
1 is extended using additional data provided by Thales. The extended dataset

2 consists of 48 radar recordings, including 2087-second recordings of UAVs and 3 322-second recordings of non-UAVs. The dataset covers a wide range of UAVs

4 such as single-rotor, multi-rotor and fixed-wing types, and non-UAVs including

5 background and targets most similar to UAVs such as birds. Hence the data

6 distribution well represents the data population for UAV detection after filtering

7 out targets obviously different from UAVs by other means. The model built on

8 the dataset could be applied in practical scenarios. The sample spectrograms of UAVs and birds are shown in Fig. 2.

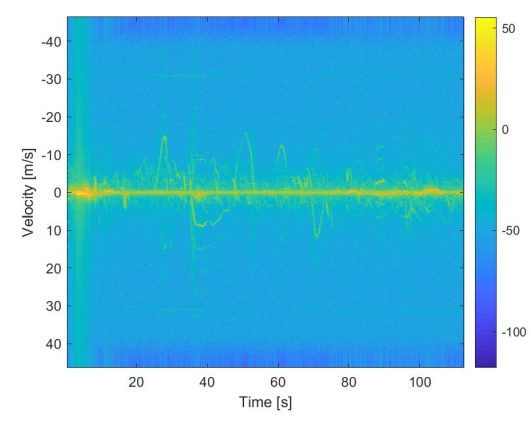

(a) Spectrogram of Easystar glider

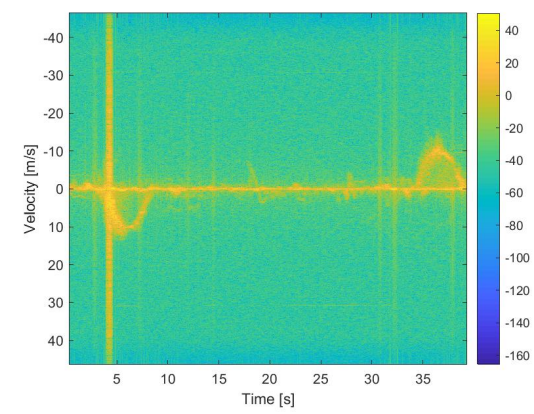

(c) Spectrogram of a bird

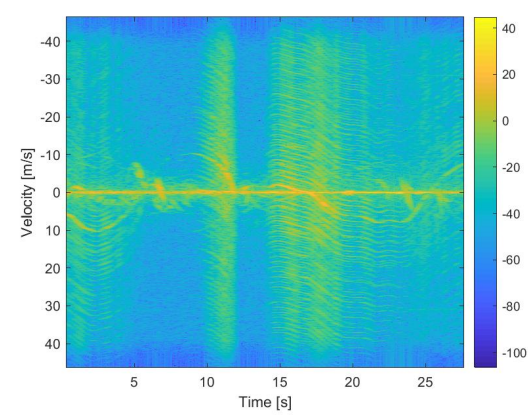

(b) Spectrogram of Trex 450 helicopter

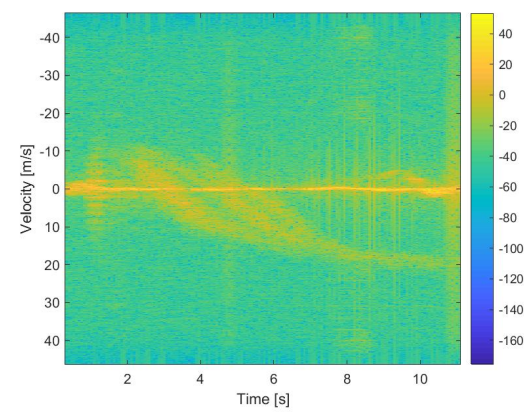

(d) Spectrogram of a group of birds

Figure 2: Sample spectrograms of UAVs and birds.

As a longer duration is needed for the dynamic time warping (DTW) [4], the recordings are chopped into 1-second samples when evaluating DTW. For other approaches, the recordings are chopped into 50-ms samples, unless otherwise 
stated. As a result, there are in total 2409 samples when evaluating DTW, and 48180 samples when evaluating others. This is a relatively large dataset for a two-class classification problem. Half of the dataset is randomly chosen as training samples, and the other half is chosen as testing samples. The experiments are repeated 10 times, and the average performance is reported.

Denote the number of UAV samples being correctly classified and falsely classified as $n_{T P}$ and $n_{F N}$, and the number of non-UAV samples being correctly classified and falsely classified as $n_{T N}$ and $n_{F P}$, respectively. The false acceptance rate (FAR) and the false rejection rate (FRR) are defined as follow:

$$
\begin{aligned}
& F A R=\frac{n_{F P}}{n_{F P}+n_{T N}}, \\
& F R R=\frac{n_{F N}}{n_{F N}+n_{T P}} .
\end{aligned}
$$

By varying the decision threshold, different combinations of FAR and FRR could be derived. When these two error rates are the same, it is defined as the equal error rate (EER). Three evaluation criteria are reported in this paper: EER, the FAR at the FRR of $1 \%$ (denoted as $F A R_{F R R=1 \%}$ ) and the FAR at the FFR of $0.1 \%$ (denoted as $F A R_{F R R=0.1 \%}$ ). These three criteria are chosen because: 1) The EER is commonly used in detection tasks. 2) To evaluate how the system performs at a low missing detection rate of UAVs (i.e., a low FRR), $F A R_{F R R=1 \%}$ and $F A R_{F R R=0.1 \%}$ are reported.

The regularized 2-D complex-log Fourier transform [17] is utilized as the initial feature representation, in which the spectrum utilizes 256 data points and the windows have $50 \%$ overlapping. After removing the clutter and some unreliable high-frequency components, the initial feature vectors have $201 \times 36=$ 7236 dimensions for 50-ms samples.

\subsection{Analysis of Data Distribution}

The distribution of the dataset is examined how far it deviates from the Gaussian. The feature vectors have 7236 dimensions. It is infeasible to visualize the data distribution in such a high-dimensional space. Thus, the first two principal components of the UAV/non-UAV class are extracted. Then, the 


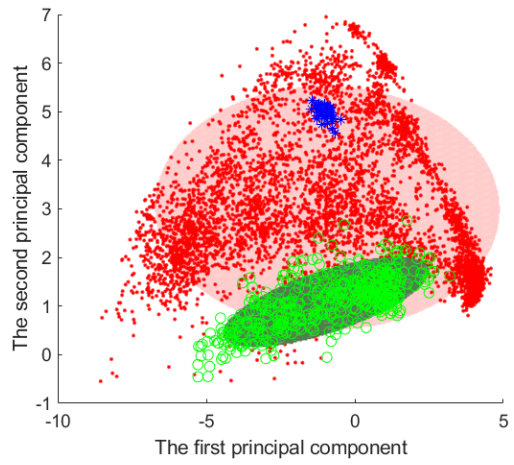

(a) 3 clusters for UAV samples

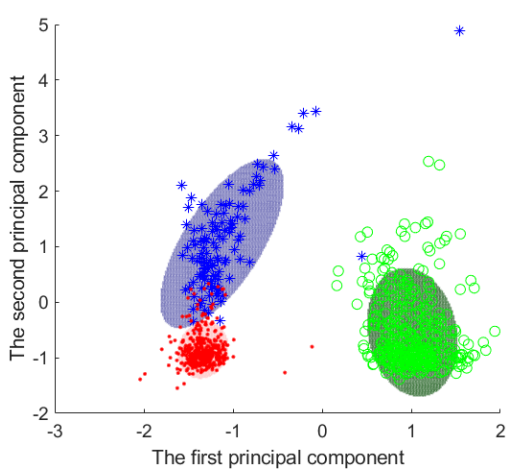

(c) 3 clusters for non-UAV samples

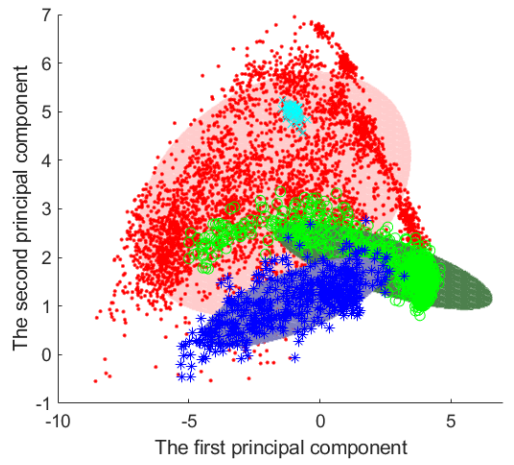

(b) 4 clusters for UAV samples

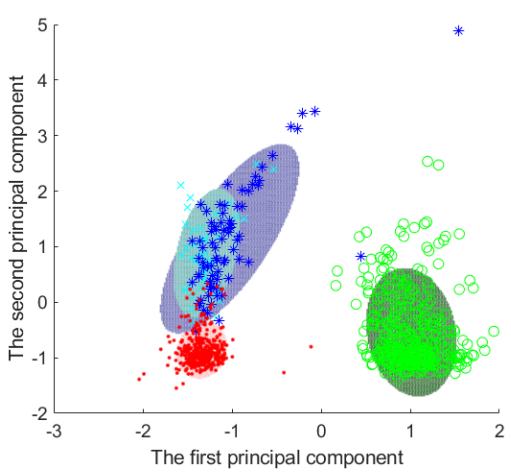

(d) 4 clusters for non-UAV samples

Figure 3: The feature dimension is 7236. The first two principal components are extracted and used to plot the distribution of UAV/non-UAV samples in the subspace built from the first two principal components, and the clustering results with 3 and 4 clusters for UAV and non-UAV classes, respectively. The figure shows that the distributions of both UAV class and non-UAV class are far away from the Gaussian.

1 clustering results using the GSC algorithm with 3 and 4 clusters for the UAV

2 class and the non-UAV class are plotted respectively in the subspace built from

3 the first two principal components, as shown in Fig. 3. Take note that the results

4 are plotted in two different subspaces, as they utilize the first two principal

5 components of UAV samples and non-UAV samples, respectively.

6 The following can be observed from the plots:

1. The distributions of both UAV and non-UAV classes are far away from 


\footnotetext{
${ }^{1}$ The matlab code of DTW can be downloaded from http://labrosa.ee.columbia.edu/ $\operatorname{matlab} / \mathrm{dtw} /$.
}

\subsection{Comparison to State-of-the-Art Approaches}

In literature, not many approaches are specifically designed for classifying UAVs from birds, except support vector machine on the integrated feature vector derived from spectrogram and cadence velocity diagram [21] and the authors' previous approach [17]. The dynamic time warping [4] and the robust principal component analysis (PCA) [7] are two recent approaches published in reputable journals, but designed for other radar-target-recognition tasks. They are hence modified for UAV detection and compared with the proposed approach.

\subsubsection{Classification Results Using Dynamic Time Warping}

The dynamic time warping ${ }^{1}[4]$ is applied on the spectrogram to align the possible time variations. The recordings are chopped into 1-second samples due to computational complexity constraints. The optimal path derived by DTW is treated as the distance between two samples. The distances from one sample to all others are treated as the feature vector. A linear support vector machine with

UAV nor non-UAV samples follow the Gaussian distribution.

2. The data distribution of the UAV class is difficult to model, as there are many different kinds of UAVs. In addition, due to the pose variations, the micro-Doppler signatures of UAVs may appear very different.

3. For the non-UAV class, there are roughly two clusters, which correspond to background samples and bird samples, two main types of non-UAVs in the current dataset.

4. For both UAV and non-UAV samples, there are some outliers, which are far away from any cluster. Both the complex data distribution and the outliers are the motivations of using the greedy subspace clustering to handle these two challenges. 
1 the cost parameter $C=40$ is trained as the classifier. The average classification errors over 10 trials are shown in Table 1.

\begin{tabular}{|l|l|l|}
\hline EER & $\boldsymbol{F} \boldsymbol{A R}_{\boldsymbol{F R R}=\mathbf{1 \%}}$ & $\boldsymbol{F} \boldsymbol{A} \boldsymbol{R}_{\boldsymbol{F R R}=\mathbf{0 . 1 \%}}$ \\
\hline $18.69 \%$ & $92.93 \%$ & $99.36 \%$ \\
\hline
\end{tabular}

Table 1: Classification errors using the dynamic time warping [4].

5 is expected to be low, the false acceptance rate is very high, as high as $92.93 \%$.

6 7.3.2. Classification Results Using Robust PCA

7 The same procedures as in $[7,17]$ are utilized to implement the robust PCA.

8 The feature vector is obtained by averaging the spectrogram over time. The 9 minimum covariance determinant (MCD) estimator implemented using "rrcov" 10 package in $\mathrm{R}$ programming is used to remove the outliers. PCA is then used to 11 reduce the feature dimensionality. Finally, the feature vectors are normalized to

12 zero mean with unit variance, and classified by a linear support vector machine PCA performs better than the DTW.

\begin{tabular}{|l|l|l|}
\hline EER & $\boldsymbol{F A R}_{\boldsymbol{F R R}=\mathbf{1 \%}}$ & $\boldsymbol{F} \boldsymbol{A} \boldsymbol{R}_{\boldsymbol{F R R}=\mathbf{0 . 1 \%}}$ \\
\hline $10.15 \%$ & $53.41 \%$ & $81.34 \%$ \\
\hline
\end{tabular}

Table 2: Classification performance using the robust PCA [7]. 


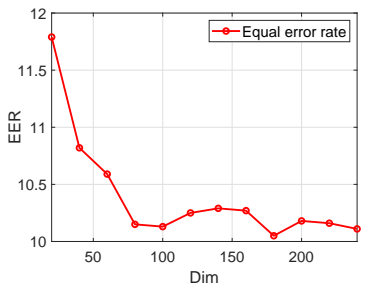

(a) EER.

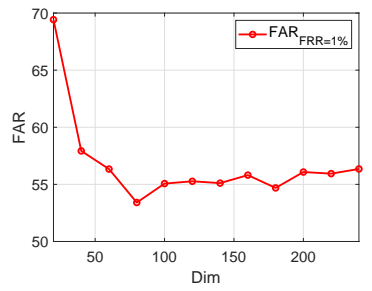

(b) $F A R_{F R R=1 \%}$.

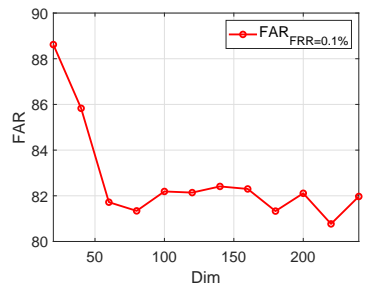

(c) $F A R_{F R R=0.1 \%}$

Figure 4: Error rates vs. feature dimensionalities for the robust PCA [7].

1 7.3.3. Support Vector Machine on Spectrogram and Cadence Velocity Diagram

A support vector machine was applied on spectrogram and cadence velocity 3 diagram to differentiate UAVs from birds [21]. The implementation described 4 in [21] is strictly followed. The feature vector consists of three parts: 1) The 5 average spectrogram over time; 2) The first left singular vector of the spec6 trogram after the Singular Value Decomposition on the spectrogram; 3) The 7 average cadence velocity diagram over cadence frequency. These three are then 8 concatenated as the final feature vector and classified by a linear support vector 9 machine with the cost parameter $C=40$. This approach is named as SVM-S-

10 CVD. The classification results are shown in Table 3. SVM-S-CVD performs better than DTW and RPCA, as it utilizes both spectrogram and CVD.

\begin{tabular}{|l|l|l|}
\hline EER & $\boldsymbol{F} \boldsymbol{A R}_{\boldsymbol{F R} \mathbf{R}=\mathbf{1 \%}}$ & $\boldsymbol{F} \boldsymbol{A} \boldsymbol{R}_{\boldsymbol{F R} \mathbf{R}=\mathbf{0 . 1 \%}}$ \\
\hline $7.46 \%$ & $41.30 \%$ & $90.24 \%$ \\
\hline
\end{tabular}

Table 3: The error rates for a support vector machine on the integrated feature vector extracted from spectrogram and cadence velocity diagram [21].

\section{7.3.4. Performance Evaluation of Proposed Approach}

13 Now the proposed approach (greedy subspace clustering, multi-Gaussian 14 subspace reliability analysis and subspace-fusion scheme, denoted as GSC-MGSRA-

$15 \mathrm{SF}$ ) is compared with the subspace reliability analysis based on the uni-Gaussian 16 model (denoted as SRA) [17]. Both utilize the robust spectral analysis in Sec- 
1 tion 3 to extract the initial feature representation. In addition, GMM-MGSRA-

2 $\mathrm{SF}$ is implemented to evaluate the performance gain over the GMM, where GSC

3 is replaced by the Gaussian mixture model and the rest procedures are the same

4 as the proposed approach.

5 SRA: The subspace reliability analysis is applied on two covariance matrices

6 separately in two different subspaces, and a ratio test is employed to differentiate

$7 \mathrm{UAVs}$ from non-UAVs. The dimensionality of these two subspaces is reduced 8 using SRA to $\{1,2, \ldots, 10,20,30, \ldots, 200\}$, respectively, and the performance 9 at the optimal combination of these two dimensionalities is reported.

10 Proposed GSC-MGSRA-SF: As SRA is evaluated for at most 200 dimen${ }_{11}$ sions, the principal component analysis is applied to reduce the dimensionality 12 from 7236 to 200 for a fair comparison. Then, the greedy subspace clustering 13 is applied on the UAV class and the non-UAV class, respectively. To evaluate 14 how the performance varies with the number of clusters, the number of clusters 15 is explicitly chosen as 5, 10 and 20 for both classes. For the nearest-subspace16 neighbor algorithm [36], the number of nearest neighbors is set as $40^{2}$ and the 17 number of feature dimensions of the linear subspace is set to the default value ${ }_{18} 20$. To remove the outliers that may form a cluster, the samples in the smallest cluster for each class are removed.

The Mahalanobis distances at dimensions of $\{1,2, \ldots, 10,20,30, \ldots, 200\}$ 21 are evaluated for both UAV and non-UAV classes. These dimensionalities are well spread across the possible optimal dimensionality within 200 . These distances are treated as the feature vector and classified by a linear support vector machine. The cost parameter for the SVM is explicitly set to 20. The proposed approach is denoted as GSC-MGSRA-SF- $M$, where $M$ denotes the number of clusters used.

GMM-MGSRA-SF: To show the performance gain against the Gaussian mix-

\footnotetext{
${ }^{2}$ The default value is 20 . As there are thousands of samples, the similarity matrix is large. It will lead to a very sparse similarity matrix if utilizing only 20 nearest neighbors, and lead to numerical instability for the subsequent spectral clustering. Thus it increases to 40 .
} 
1 ture model, the GSC algorithm is replaced by GMM and the rest procedures are

2 kept the same as the proposed approach. Due to the curse of dimensionality,

3 the same diagonal matrix is used for all clusters of each class when building the

4 mixture model using the EM algorithm. The number of mixture components is set to 5 for each class. The experimental results are summarized in Table 4.

\begin{tabular}{|l|l|l|l|}
\hline Method & EER & $\boldsymbol{F ~ A ~}_{\boldsymbol{F R R = 1 \%}}$ & $\boldsymbol{F} \boldsymbol{A R}_{\boldsymbol{F R \boldsymbol { R }}=\mathbf{0 . 1} \%}$ \\
\hline SRA [17] & $5.56 \%$ & $25.20 \%$ & $44.80 \%$ \\
\hline GMM-MGSRA-SF & $4.76 \%$ & $16.15 \%$ & $43.52 \%$ \\
\hline GSC-MGSRA-SF-5 & $3.95 \%$ & $14.50 \%$ & $44.37 \%$ \\
\hline GSC-MGSRA-SF-10 & $3.13 \%$ & $7.91 \%$ & $40.77 \%$ \\
\hline GSC-MGSRA-SF-20 & $\mathbf{3 . 0 5 \%}$ & $\mathbf{7 . 5 5 \%}$ & $\mathbf{3 0 . 0 1 \%}$ \\
\hline
\end{tabular}

Table 4: Comparison to SRA and GMM-MGSRA-SF, and evaluation of the proposed approach on different number of clusters used in the greedy subspace clustering algorithm.

7 SF outperforms SRA, which shows the advantages of modeling the complex

8 data distribution as the multi-Gaussian model over the uni-Gaussian one. The

9 proposed approach also outperforms GMM-MGSRA-SF, which shows the effectiveness of the GSC algorithm over the Gaussian mixture model. In general, the error rates decrease with the increase of the number of clusters used in GSC-MGSRA-SF. The multi-Gaussian model better models the complex data distribution by using more clusters. The performance gain becomes marginal when the number of clusters is large. When more clusters are used, the number of samples falling into each cluster becomes smaller. Thus, the distribution of each cluster may not be well estimated using a limited number of samples. As a result, the performance gain is small, or the performance may even drop if the number of clusters increases further. For the rest of experiments, the number of clusters is set to 20 .

The performance comparisons to the state-of-the-art approaches are summarized in Table 5. The proposed approach significantly outperforms the others. 


\begin{tabular}{|c|c|c|c|}
\hline Method & EER & $F A R_{F R R=1 \%}$ & $F A R_{F R R=0.1 \%}$ \\
\hline DTW [4] & $18.69 \%$ & $92.93 \%$ & $99.36 \%$ \\
\hline RPCA [7] & $10.15 \%$ & $53.41 \%$ & $81.34 \%$ \\
\hline SVM-S-CVD [21] & $7.46 \%$ & $41.30 \%$ & $90.24 \%$ \\
\hline SRA [17] & $5.56 \%$ & $25.20 \%$ & $44.80 \%$ \\
\hline GMM-MGSRA-SF & $4.76 \%$ & $16.15 \%$ & $43.52 \%$ \\
\hline Proposed GSC-MGSRA-SF & $3.05 \%$ & $7.55 \%$ & $30.01 \%$ \\
\hline
\end{tabular}

Table 5: Summary of the comparisons to the state-of-the-art approaches.

\section{7.4. Performance Evaluation on Noise Robustness}

$3 \quad$ Noise is injected into the radar return signal to evaluate the noise robustness

4 of the proposed approach. The signal-to-noise ratio (SNR) is calculated as:

$$
S N R=10 \log _{10}\left(\frac{P_{\boldsymbol{x}}}{P_{\boldsymbol{n}}}\right),
$$

where $P_{\boldsymbol{n}}$ is the power of the injected Gaussian noise and $P_{\boldsymbol{x}}$ is the power of the radar signal after removing the clutter. Gaussian noise is used as it is one of

the most common noise types. The clutter is removed before injecting the noise

10 as it is not relevant to the radar target but much stronger than the Doppler

11 signatures. Note that the main body Doppler is much stronger than the micro-

12 Doppler signatures. Thus, the actual SNR w.r.t. $\mathrm{mDS}$ is much lower than the 13 reported SNR. The error rates for different SNRs are summarized in Table 6.

Table 6 shows that when the noise is small or even comparable to the micro15 Doppler signatures, the proposed approach achieves a fairly good performance.

16 The error rates do not change significantly when the noise level is low. The

${ }_{17}$ proposed approach is shown robust to noise. Even when the noise level is high,

18 the error rates of the proposed approach remain at a reasonable level.

19 7.5. Performance Evaluation by Varying Observation Durations

Here, the proposed approach is evaluated for various observation durations.

${ }_{21}$ Intuitively, if there is a longer observation duration, more information about the 


\begin{tabular}{|l|l|l|l|}
\hline SNR & EER & $\boldsymbol{F A R}_{\boldsymbol{F R R}=\mathbf{1 \%}}$ & $\boldsymbol{F A R}_{\boldsymbol{F R R}=\mathbf{0 . 1 \%}}$ \\
\hline-10 & $12.79 \%$ & $39.12 \%$ & $66.35 \%$ \\
\hline 0 & $5.87 \%$ & $21.56 \%$ & $57.79 \%$ \\
\hline 10 & $3.37 \%$ & $8.39 \%$ & $47.35 \%$ \\
\hline 20 & $3.10 \%$ & $8.10 \%$ & $51.81 \%$ \\
\hline clean & $3.05 \%$ & $7.55 \%$ & $30.01 \%$ \\
\hline
\end{tabular}

Table 6: The classification errors vs. different SNRs. The proposed approach is robust to noise to a large extent.

radar target can be obtained, and hence a higher classification accuracy can be 2 achieved, but the extracted feature vector will become larger.

3 In the previous experiments, the observation duration is set as $50 \mathrm{~ms}$, as 4 suggested by Thales. In this experiment, the system is evaluated for the obser5 vation durations of 10, 25, 50, 100 and $200 \mathrm{~ms}$. Table 7 summarizes the initial 6 feature dimensionality and the number of samples for different observation du7 rations. If the number of samples is large, the NSN algorithm used in the greedy 8 subspace clustering will take a long time to execute as it needs to loop through 9 all samples to find the nearest subspace neighbors, and the subsequent spectral 10 clustering requires a large amount of memory and a long execution time. On the 11 other hand, if the initial feature dimensionality is large, the covariance matrix 12 of the initial feature vector will be large and require a huge amount of memory.

The error rates and the average execution time of one sample vs. the ob15 servation durations are shown in Table 8. The proposed approach is trained 16 and tested on a Dell PC with Intel Xeon Silver 4108 CPU @1.80 GHz. The 17 experimental results are consistent with the previous analysis, i.e., longer the 18 observation window, better the classification performance. When the duration 19 is very short, e.g., $10 \mathrm{~ms}$, the classification errors significantly increase because 20 not even one full rotation cycle of the rotor blade of a UAV could be captured in ${ }_{21}$ such a short time. The intra-class variations of the UAV class greatly increase, 


\begin{tabular}{|l|l|l|}
\hline $\begin{array}{l}\text { Observation } \\
\text { duration }(\mathbf{m s})\end{array}$ & $\begin{array}{l}\text { Initial feature } \\
\text { dimensionality }\end{array}$ & $\begin{array}{l}\text { The number } \\
\text { of samples }\end{array}$ \\
\hline 10 & 1,206 & 240,900 \\
\hline 25 & 3,417 & 96,360 \\
\hline 50 & 7,236 & 48,180 \\
\hline 100 & 14,874 & 24,090 \\
\hline 200 & 29,949 & 12,045 \\
\hline
\end{tabular}

Table 7: The initial feature dimensionality and the number of samples vs. the observation durations. If the duration is long, the feature dimensionality will be large. On the other hand, if the duration is short, the number of samples will be large.

1 and hence the error rates significantly increase. On the other hand, when the 2 observation duration is sufficiently long, the error rates do not significantly de3 crease with a further increase of the duration, e.g., the performance gain from $4100 \mathrm{~ms}$ to $200 \mathrm{~ms}$ is not as significant as others. In terms of execution time, 5 although the proposed approach looks complicated, it could predict one sample in real time.

\begin{tabular}{|l|l|l|l|l|}
\hline $\begin{array}{l}\text { Observation } \\
\text { duration }(\mathbf{m s})\end{array}$ & EER & $\boldsymbol{F A R _ { F R R = 1 \% }}$ & $\boldsymbol{F A R _ { F R R = 0 . 1 \% }}$ & $\begin{array}{l}\text { Execution } \\
\text { time (ms) }\end{array}$ \\
\hline 10 & $8.45 \%$ & $37.87 \%$ & $65.39 \%$ & 3.61 \\
\hline 25 & $4.20 \%$ & $15.08 \%$ & $56.42 \%$ & 4.06 \\
\hline 50 & $3.05 \%$ & $7.55 \%$ & $30.01 \%$ & 4.76 \\
\hline 100 & $2.02 \%$ & $3.50 \%$ & $22.57 \%$ & 7.99 \\
\hline 200 & $\mathbf{1 . 7 2 \%}$ & $\mathbf{2 . 3 1 \%}$ & $\mathbf{1 3 . 4 1 \%}$ & 20.81 \\
\hline
\end{tabular}

Table 8: Classification error rates and average prediction time of one test sample for different observation durations.

\section{7.6. Demo of UAV Detection}

A live demo for UAV detection is implemented using Matlab. The demo

9 GUI is shown in Fig. 5. The sub-figure shown at the top is the spectrogram of 
1 the radar signal. The sub-figure on the bottom-left is the video capture of the

2 target and the real-time classification result for the current sample of $50 \mathrm{~ms}$ is

3 shown on the right. The cumulative classification results as UAVs or non-UAVs,

4 and the classification time for the current sample are shown in bottom-middle.

5 Once the demo starts execution, the user will be prompted to choose a radar

6 recording for analysis. The overlapping ratio of $50 \%$ is preset in the demo, as

7 the model needs to be re-trained if the key parameters change. The overlapping

8 ratio controls the time resolution of the spectrogram. Larger overlapping ratio

9 means higher resolution and hence higher classification accuracy at a cost of

10 higher computational complexity. For a speed-accuracy trade-off, it is set to

${ }_{11} 50 \%$. It takes about $15.33 \mathrm{~ms}$ to classify a sample using Matlab 2019a, on a

12 Dell PC with Intel Xeon Silver 4108 CPU @1.80 GHz. This demo shows that the proposed system can detect UAVs reliably in real time.
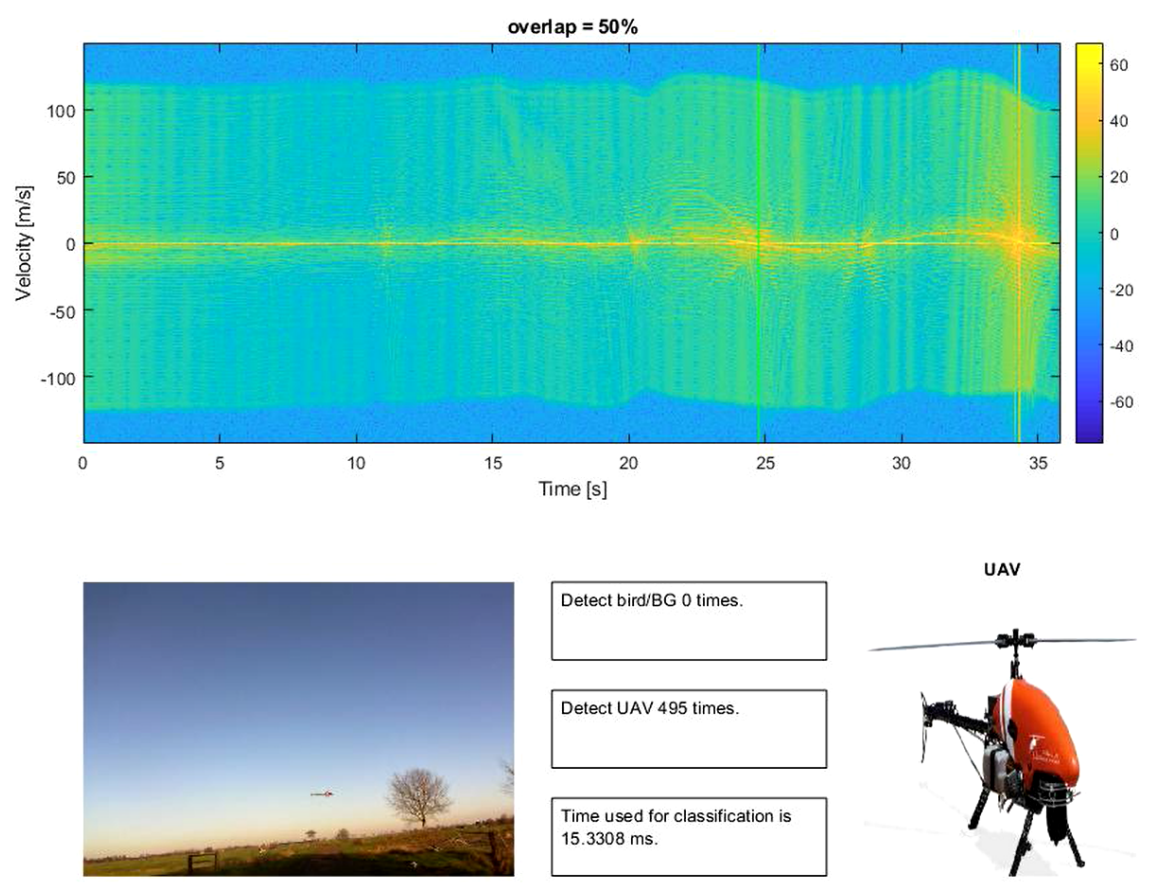

Figure 5: GUI of the UAV-detection demo. It shows that the proposed system could detect UAVs reliably in real time. 

30 radar

In this paper, a three-step classification framework is proposed to address three challenges in radar UAV detection: outliers in the data, complex data distribution and the curse of dimensionality. In the first step, the authors propose to utilize the greedy subspace clustering to handle the outliers and model the complex data distribution. The expectation-maximization algorithm to derive the Gaussian mixture model could not well cluster the samples due to the curse of dimensionality. To circumvent this problem, a multi-Gaussian subspace reliability analysis is proposed in the second step to handle the unreliable feature dimensions of the derived multi-Gaussian model. In the third step, a subspace-fusion scheme is proposed to combine the distances of a sample to different clusters of different classes at different dimensionalities. The proposed system is compared with existing approaches on a large benchmark dataset, and significantly outperforms the state-of-the-art approaches.

The proposed three-step classification framework could well handle the complex distribution of radar data. However, a potential problem here is that the model in the early stage is optimized without considering the later ones. The future plan is to integrate these three steps as one unified algorithm, e.g., considering the reliability of newly added subspace during the GSC algorithm. The second potential research direction is to integrate the proposed subspace fusion with other subspace approaches, where the optimal dimension is difficult to determine or a single optimal dimension is not sufficient. Thirdly, it is still an open question how to optimally model the complex data distribution. The proposed framework demonstrates the effectiveness of the multi-Gaussian model. The plan is to explore other ways to construct the model, or extend this research to other pattern-recognition tasks, e.g., from UAV detection to UAV classification. Lastly, as a new dataset has been collected using SQUIRE radar (a FMCW radar) from Thales, the authors will explore the feasibility of not only detecting UAV, but also determining the direction and the distance of the UAV to the 


\section{Acknowledgment}

This research is supported in part by Singapore Future Systems and Technology Directorate (FSTD) under project reference MINDEF-NTU-DIRP/2014/01, in part by Singapore Ministry of Education Academic Research Fund Tier 1 RG 123/15, and in part by the Ningbo Municipal Bureau Science and Technology under Grant 2017D10034 and Grant 2019B10026. Thank Thales Solutions Asia for providing the data. Special thanks to Mr. Adriaan Smits, the Director of Centre of Excellence for Radar \& Integrated Sensors, Thales Solutions Asia, for the valuable discussion and advice.

\section{References}

[1] Commercial UAV market analysis by product (fixed wing, rotary blade, nano, hybrid), by application (agriculture, energy, government, media \& entertainment) and segment forecasts to 2022, https://www.grandviewresearch.com/industry-analysis/ commercial-uav-market, 2016.

[2] Y. Pan, B. Bhargava, Z. Ning, N. Slavov, S. Li, J. Liu, S. Xu, C. Li, T. Zhu, Safe and efficient UAV navigation near an airport, in: IEEE International Conference on Communications (ICC), 1-6, 2019.

[3] M. Ritchie, F. Fioranelli, H. Griffiths, B. Torvik, Micro-drone RCS analysis, in: IEEE Radar Conference, IEEE, 452-456, 2015.

[4] G. E. Smith, K. Woodbridge, C. J. Baker, Radar micro-Doppler signature classification using dynamic time warping, IEEE Transactions on Aerospace and Electronic Systems 46 (3) (2010) 1078-1096.

[5] Y. Wang, Q. Liu, A. E. Fathy, CW and pulse-Doppler radar processing based on FPGA for human sensing applications, IEEE Transactions on Geoscience and Remote Sensing 51 (5) (2013) 3097-3107.

[6] P. Molchanov, K. Egiazarian, J. Astola, R. I. A. Harmanny, J. J. M. de Wit, Classification of small UAVs and birds by micro-Doppler signatures, in: European Radar Conference, $172-175,2013$.

[7] J. Zabalza, C. Clemente, G. Di Caterina, J. Ren, J. J. Soraghan, S. Marshall, Robust PCA for micro-Doppler classification using SVM on embedded systems, IEEE Transactions on Aerospace and Electronic Systems 50 (3) (2014) 2304-2310. 
[8] J. Park, J. T. Johnson, N. Majurec, M. Frankford, K. Stewart, G. E. Smith, L. Westbrook, Simulation and analysis of polarimetric radar signatures of human gaits, IEEE Transactions on Aerospace and Electronic Systems 50 (3) (2014) 2164-2175.

[9] J. Ren, X. Jiang, Radar micro-Doppler signature analysis and its application on gait recognition, in: Third International Workshop on Pattern Recognition, vol. 10828, International Society for Optics and Photonics, 1082803, 2018.

[10] Y. Yang, C. Hou, Y. Lang, D. Guan, D. Huang, J. Xu, Open-set human activity recognition based on micro-Doppler signatures, Pattern Recognition 85 (2019) 60 - 69.

[11] A. Huizing, M. Heiligers, B. Dekker, J. de Wit, L. Cifola, R. Harmanny, Deep learning for classification of mini-UAVs using micro-Doppler spectrograms in cognitive radar, IEEE Aerospace and Electronic Systems Magazine 34 (11) (2019) 46-56.

[12] R. Harmanny, J. de Wit, G. Prémel-Cabic, Radar micro-Doppler feature extraction using the spectrogram and the cepstrogram, in: European Radar Conference, IEEE, 165-168, 2014.

[13] S. Björklund, T. Johansson, H. Petersson, Evaluation of a micro-Doppler classification 
[19] Y. Zhao, Y. Su, The extraction of micro-Doppler signal with EMD algorithm for radarbased small UAVs' detection, IEEE Transactions on Instrumentation and Measurement 69 (3) (2020) 929-940.

[20] B. K. Kim, H.-S. Kang, S.-O. Park, Drone classification using convolutional neural networks with merged Doppler images, IEEE Geoscience and Remote Sensing Letters 14 (1) (2016) 38-42.

[21] L. Fuhrmann, O. Biallawons, J. Klare, R. Panhuber, R. Klenke, J. Ender, Micro-Doppler analysis and classification of UAVs at Ka band, in: 18th International Radar Symposium (IRS), 1-9, 2017.

[22] J. S. Patel, C. Al-Ameri, F. Fioranelli, D. Anderson, Multi-time frequency analysis and classification of a micro-drone carrying payloads using multistatic radar, The Journal of Engineering 2019 (20) (2019) 7047-7051.

[23] J. Chen, L. Du, H. He, Y. Guo, Convolutional factor analysis model with application to radar automatic target recognition, Pattern Recognition 87 (2019) 140 - 156.

[24] B. Feng, B. Chen, H. Liu, Radar HRRP target recognition with deep networks, Pattern Recognition 61 (2017) 379 - 393.

[25] X. Chen, J. Guan, Z. Bao, Y. He, Detection and extraction of target with micromotion in spiky sea clutter via short-time fractional Fourier transform, IEEE Transactions on Geoscience and Remote Sensing 52 (2) (2014) 1002-1018.

[26] Y. Li, L. Du, H. Liu, Hierarchical classification of moving vehicles based on empirical mode decomposition of micro-Doppler signatures, IEEE Transactions on Geoscience and Remote Sensing 51 (5) (2013) 3001-3013.

[27] A. Alipour-Fanid, M. Dabaghchian, N. Wang, P. Wang, L. Zhao, K. Zeng, Machine learning-based delay-aware UAV detection and operation mode identification over encrypted Wi-Fi traffic, IEEE Transactions on Information Forensics and Security 15 (2020) $2346-2360$.

[28] N. Regev, I. Y. Iofedov, D. Wulich, Classification of single and multi propelled miniature drones using multilayer perceptron artificial neural network, in: International Conference on Radar Systems (Radar 2017), 1-5, 2017.

[29] J. Ren, X. Jiang, J. Yuan, A Chi-squared-transformed subspace of LBP histogram for visual recognition, IEEE Transactions on Image Processing 24 (6) (2015) 1893-1904.

[30] F. Zhao, Y. Liu, K. Huo, S. Zhang, Z. Zhang, Radar HRRP target recognition based on stacked autoencoder and extreme learning machine, Sensors 18 (1) (2018) 173. 
[31] G. Dong, G. Kuang, N. Wang, L. Zhao, J. Lu, SAR target recognition via joint sparse representation of monogenic signal, IEEE Journal of Selected Topics in Applied Earth Observations and Remote Sensing 8 (7) (2015) 3316-3328.

[32] W. Liu, J. Yuan, G. Zhang, Q. Shen, HRRP target recognition based on kernel joint discriminant analysis, Journal of Systems Engineering and Electronics 30 (4) (2019) $703-708$.

[33] L. Li, Z. Liu, T. Li, Radar high-resolution range profile feature extraction method based on multiple kernel projection subspace fusion, IET Radar, Sonar Navigation 12 (4) (2018) $417-425$.

[34] H. Chen, W. Wang, X. Feng, Structured sparse subspace clustering with within-cluster grouping, Pattern Recognition 83 (2018) 107 - 118.

[35] J. Yang, J. Liang, K. Wang, P. L. Rosin, M. Yang, Subspace clustering via good neighbors, IEEE Transactions on Pattern Analysis and Machine Intelligence 42 (6) (2020) 15371544 .

[36] D. Park, C. Caramanis, S. Sanghavi, Greedy subspace clustering, in: Advances in Neural Information Processing Systems, 2753-2761, 2014.

[37] C. Premebida, U. Nunes, A multi-target tracking and GMM-classifier for intelligent vehicles, in: IEEE Intelligent Transportation Systems Conference, IEEE, 313-318, 2006.

[38] J. Yu, C. Chaomurilige, M.-S. Yang, On convergence and parameter selection of the EM and DA-EM algorithms for Gaussian mixtures, Pattern Recognition 77 (2018) 188 - 203.

[39] J. Ma, X. Jiang, J. Jiang, Y. Gao, Feature-guided Gaussian mixture model for image matching, Pattern Recognition 92 (2019) $231-245$.

[40] X. Jiang, Linear subspace learning-based dimensionality reduction, Signal Processing Magazine, IEEE 28 (2) (2011) 16-26.

[41] X. Jiang, B. Mandal, A. Kot, Eigenfeature regularization and extraction in face recognition, IEEE Transactions on Pattern Analysis and Machine Intelligence 30 (3) (2008) $383-394$.

[42] X. Jiang, Asymmetric principal component and discriminant analyses for pattern classification, IEEE Transactions on Pattern Analysis and Machine Intelligence 31 (5) (2009) $931-937$.

[43] J. Ren, X. Jiang, J. Yuan, A complete and fully automated face verification system on mobile devices, Pattern Recognition 46 (1) (2013) 45-56. 
1 [44] X. Wang, X. Jiang, J. Ren, Blood vessel segmentation from fundus image by a cascade classification framework, Pattern Recognition 88 (2019) 331-341.

3 [45] J. Lipor, L. Balzano, Clustering quality metrics for subspace clustering, Pattern Recognition 104 (2020) 107328. 